\title{
Dietary patterns associated with overweight and obesity among Brazilian schoolchildren: an approach based on the time-of-day of eating events
}

\author{
Emil Kupek ${ }^{1 *}$, Adriana S. Lobo ${ }^{2}$, Danielle B. Leal ${ }^{2}$, France Bellisle ${ }^{3}$ and Maria Alice A. de Assis ${ }^{2}$ \\ ${ }^{1}$ Department of Public Health, Center of Health Science, Federal University of Santa Catarina, Campus Universitário - \\ Trindade, Florianópolis 88040-900, Brazil \\ ${ }^{2}$ Center of Health Science, Federal University of Santa Catarina, Campus Universitário - Trindade, Florianópolis \\ 88040-900, Brazil \\ ${ }^{3}$ Equipe de Recherche en Epidémiologie Nutritionnelle, Centre de Recherche en Epidémiologie et Statistiques, Université \\ Paris 13, Inserm (U1153), Inra (U1125), Cnam, COMUE Sorbonne Paris Cité, Bobigny 93017, France
}

(Submitted 11 May 2016 - Final revision received 23 October 2016 - Accepted 3 November 2016 - First published online 15 December 2016)

\begin{abstract}
Several studies reported that the timing of eating events has critical implications in the prevention of obesity, but dietary patterns regarding the time-of-day have not been explored in children. The aim of this study was to derive latent food patterns of daily eating events and to examine their associations with overweight/obesity among schoolchildren. A population-based cross-sectional study was conducted with 7-10-year-old Brazilian schoolchildren ( $n$ 1232) who completed the Previous Day Food Questionnaire, illustrated with twenty-one foods/beverages in six daily eating events. Latent class analysis was used to derive dietary patterns whose association with child weight status was evaluated by multivariate multinomial regression. Four mutually exclusive latent classes of dietary patterns were identified and labelled according to the time-of-day of eating events and food intake probability (FIP): (A) higher FIP only at lunch; (B) lower FIP at all eating events; (C) higher FIP at lunch, afternoon and evening snacks; (D) lower FIP at breakfast and at evening snack, higher FIP at other meals/snacks. The percentages of children within these classes were $32 \cdot 3,48 \cdot 6,15 \cdot 1$ and $4 \cdot 0 \%$, respectively. After controlling for potential confounders, the mean probabilities of obesity for these classes were $6 \%(95 \%$ CI $3 \cdot 0,9 \cdot 0), 13 \%(95 \%$ CI $9 \cdot 0,17 \cdot 0), 12 \%(95 \%$ CI $6 \cdot 0,19)$ and $11 \%(95 \%$ CI 5.0, 17.0), in the same order. In conclusion, the children eating traditional lunch with rice and beans as the main meal of the day (class A) had the lowest obesity risk, thus reinforcing the importance of both the food type and the time-of-day of its intake for weight status.
\end{abstract}

Key words: Children's dietary patterns: Time-of-day: Eating events: Latent class analysis: Childhood obesity

For over a decade, repeated calls have been made to substitute individual foods and nutrients by dietary patterns (DP) analysis as tentative predictors of overweight and obesity, and related diseases $^{(1)}$. The rationale is that such patterns can summarise potentially synergistic effects of foods or nutrients on overweight and obesity ${ }^{(2)}$. To this end, a variety of statistical techniques such as principal component and factor analysis, cluster and latent class analysis (LCA) and reduced rank regression have been used to identify DP in children and adolescents ${ }^{(2-9)}$. A systematic review indicated that DP that are high in energy-dense, high-fat and low-fibre foods predispose young people to later overweight and obesity ${ }^{(2)}$. The incorporation of timing of food intake into DP analysis has been rarely explored in adults ${ }^{(10,11)}$. In addition, there are no studies addressing the associations between temporal DP and body-weight status of children.

In parallel with increasingly sophisticated statistical derivations of DP, their a priori definitions (e.g. distribution of energy intake across the eating occasions, consuming $v$. skipping breakfast) have also been verified as potential causes of overweight and obesity. Two major lines of research have been established to better understand the role of daily eating patterns as important drivers of the obesity epidemic. The first one focuses on the influence of daily number of eating events (meals compared with snacks) and the other one on the effect of the temporal distribution of dietary intake on diet quality and adiposity status. Neither of these lines provided consistent findings from crosssectional analyses of the relationship between children and adolescents' weight status and daily eating patterns ${ }^{(12-18)}$. Methodological limitations associated with dietary assessment methods, misreporting of energy intake and the lack of consensus of what constitutes a snack, a meal or an eating event are potential confounders ${ }^{(19)}$

The studies with an objective definition of meals and snacks showed that increased frequencies of eating events are associated

Abbreviations: DP, dietary patterns; FIP, food intake probability; LCA, latent class analysis; PDFQ-3, third version of the Previous Day Food Questionnaire. 
with higher total daily energy intake for both children and adolescents $^{(15-18)}$. Higher frequencies of snacks are linked to unhealthy dietary intake patterns in UK, US and Mexican children $^{(12-14)}$, as well as with a decrease in the diet quality index for UK adolescents when low-energy snacks were excluded from the analysis ${ }^{(15)}$. Studies conducted with children and adolescents in the UK showed no associations between the risk of overweight and/or obesity and evening meal timing, energy or nutrient intake $^{(16)}$, or daily number of eating occasions ${ }^{(12)}$. Conversely, some studies conducted in US children and adolescents found that increased eating occasions and snacking were positively associated with adiposity measures after adjustment for misreporting of energy intake ${ }^{(13)}$. In addition, an increased energy intake in the time period 18.00-19.59 hours compared with 16.00-17.59 hours was positively associated with overweight in 6-11-year-olds but negatively associated with overweight in 12-18-year-olds ${ }^{(17)}$. Higher adiposity measures for Brazilian adolescents were reported in boys (but not in girls) who skipped breakfast and replaced the traditional lunch and dinner by high-energy-dense foods ${ }^{(18)}$

Several studies on the temporal distribution of dietary intake in children and adults ${ }^{(20-28)}$ showed that the moment of intake is a crucial factor affecting food choices, satiation, satiety and other aspects of appetite. The temporal distribution of specific foods and macronutrients throughout the 24-h day have critical implications in the prevention of obesity, weight loss and regain, insulin resistance and the metabolic syndrome ${ }^{(25)}$. In addition, the association between intake timing and total daily food intake appears to be macronutrient specific in US adults $^{(22)}$. A central circadian clock is a key synchroniser of peripheral circadian clocks in the liver, intestine, pancreas and peripheral fat tissues ${ }^{(25)}$. The timing of food intake along with sleeping time, light/dark conditions, locomotor activity and energy expenditure are natural synchronisers of these clocks ${ }^{(25)}$. An unusual feeding time or disturbed eating patterns (e.g. large dinner or evening energy intake relative to breakfast or morning intake ${ }^{(20,25)}$, the night-eating syndrome ${ }^{(25)}$ and skipping breakfast ${ }^{(28)}$ ) may trigger so-called chronodisruptions, which diminish the synchronisation between external cues and internal physiological processes ${ }^{(25)}$.

Meal and snack DP based on the time-of-day of food intake have not been derived with respect to schoolchildren's weight status categories. The aim of the present study was to derive latent food patterns of daily eating events using LCA and to examine their associations with overweight and obesity among Brazilian schoolchildren.

\section{Methods}

\section{Study design and participants}

A cross-sectional survey to investigate the prevalence of overweight and obesity and related behaviours in schoolchildren was conducted in Florianopolis (Brazil) from April to October 2007. The sample size of 1100 children was estimated for this study, assuming the $22.1 \%$ prevalence of overweight with $\pm 2 \%$ precision and taking into account the stratification between public and private schools, as well as the effects of children clustering within schools. As a $10 \%$ non-response rate was expected, the targeted sample size increased to 1210 children. The schools participating in this study were selected using the same stratified cluster sampling design applied in a previous survey, with a representative sample of 7-10-year-old schoolchildren from elementary schools in $2002^{(29)}$. Of the 122 schools, nine public and seven private schools were randomly selected within four strata, defined by crossing school localisation (city centre $v$. beach area) and school type (public $v$. private). The same schools were invited to participate in the same survey again in 2007. All public schools accepted the invitation, whereas two private schools did not and were replaced by two other schools from the same stratum. One more public school was included upon request by the local educational authority. Detailed sampling procedures have been described elsewhere ${ }^{(29)}$.

The target sample for each school in 2007 was proportional to the number of eligible students (2nd-5th grade) attending the school within each stratum. In the selected schools, the respondents were chosen by systematic sampling applied to a complete list of the students. The inclusion criteria were attending the 2nd-5th grades of elementary school in Florianopolis and being 7-10 years of age. The exclusion criteria were the absence of written parental consent or being absent when either anthropometric measures were taken or when the food questionnaire was applied. In the case of refusal to take part in the study by either child or his/her parent ( $n$ 115) or in the case of absence from school during the data collection ( $n$ 45), a replacement was made from the list of other schoolchildren with the same sex, age (within \pm 0.5 years), living in the same geographic area and enroled at the same school. Considering 160 non-respondents to the initial recruiting scheme, the response rate was $87 \%$. The final sample consisted of 1232 children from seventeen schools (782 children from eleven public schools and 450 children from six private schools). The data were obtained by anthropometric measurements (height, weight) and two questionnaires. The children's questionnaire gathered information about dietary data and the parents' questionnaire provided the information on mother's height, weight and educational level, as well as on family income.

This study was conducted according to the guidelines set out in the Code of Ethics of the World Medical Association (Declaration of Helsinki) and approved by the Ethical Committee of the Federal University of Santa Catarina.

\section{Dietary intake}

Dietary data were obtained using the third version of the Previous Day Food Questionnaire (PDFQ-3) ${ }^{(30,31)}$ based on a single-day recall procedure to investigate the consumption frequency of specific foods (not nutrients) as markers of (un)healthy diet. The PDFQ-3 is a four-page pictorial food questionnaire to assess dietary intake in six eating events according to the time of the day. The online Supplementary Fig. S1 shows one page of the questionnaire. The instrument was designed for application in school settings under adult supervision following a standardised protocol $^{(30)}$. The questionnaire was previously validated through direct observation of school meals ${ }^{(30)}$ and demonstrated reasonable average sensitivity (probability of correctly reporting a 
food intake) of $70.2 \%$ and an excellent average specificity (probability of not correctly reporting food intake) of $96 \cdot 2 \%{ }^{(30)}$.

\section{Definition of the time-of-day of eating events}

Children are asked to report ingested food and beverage options at six pre-determined eating events ordered chronologically according to the time-of-day (breakfast, mid-morning snack, lunch, afternoon snack, dinner and evening snack). The focus on the time of the day of food intake without specifying its exact timing has also been used in other studies ${ }^{(23,27)}$ in a way similar to the approach used in the present study. Each eating event is illustrated with twenty-one foods/beverages or food groups: bread and biscuits, chocolate milk, coffee with milk, milk, yogurt, cheese, rice, beans, pasta, beef and poultry, fish and seafood, leafy vegetables, cooked vegetables, vegetable soup, fruits, fruit juices, French fries, pizza and hamburger, sweets, salty snacks and soft drinks (see online Supplementary Table S1). Dietary intake within each eating event was marked by crossing or circling the icons representing consumed dietary items in the questionnaire. Water was not illustrated in the PDFQ-3. The foods were chosen in order to take into account the food patterns of children in this age group, including those presented in school menus and in Brazilian dietary guidelines ${ }^{(32)}$. Data were collected on various days of the week to reflect dietary intake on school days (Monday, Tuesday, Wednesday and Thursday) and a weekend day (Sunday). The frequency of food intake was defined as the number of times per day, ranging from 0 to 126 (21 foods $\times 6$ meals/snacks) and per eating event (ranging from 0 to 6 ), assuming that only one serving was consumed on each occasion.

\section{Anthropometric measurements}

Measurements of the children's weight and height were performed in each school by five trained physical education teachers, following standard techniques ${ }^{(33)}$. Theoretical and practical workshops on measurement techniques were previously held in order to standardise anthropometric measurements ${ }^{(34)}$ taken on lightly dressed barefoot children. A digital-solar 180-kg scale (Marte $^{\circledR}$, model PP; Marte Scale and Precision Equipment) was used to measure weight, and height was measured by a portable stadiometer fixed to the wall with the zero point at ground level and scale of $0.5 \mathrm{~cm}$. BMI was computed as weight ( $\mathrm{kg}$ ) divided by height squared $\left(\mathrm{m}^{2}\right)$. Weight status based on BMI $\left(\mathrm{kg} / \mathrm{m}^{2}\right)$ was classified into four groups, according to the WHO's recommended age- and sex-standardised $z$-scores ${ }^{(35)}$, as nonoverweight $(\leq+1)$, overweight $(>+1$ to $\leq+2)$ and obese $(>+2)$.

Mother's weight status was assessed by BMI computed from self-reported weight $(\mathrm{kg})$ and height $(\mathrm{m})$, and classified as thinness $\left(<18.5 \mathrm{~kg} / \mathrm{m}^{2}\right)$, normal weight $\left(18.5-24.9 \mathrm{~kg} / \mathrm{m}^{2}\right)$, overweight non-obese $\left(25-29 \cdot 9 \mathrm{~kg} / \mathrm{m}^{2}\right)$ and obese $\left(\geq 30 \mathrm{~kg} / \mathrm{m}^{2}\right)^{(36)}$.

Household monthly income was categorised into six levels with a linear increment of $\mathrm{R} \$ 500$ (US\$269), corresponding to 1.31 minimum wage at the time of the survey (minimum wage $=$ US\$2 $24 \cdot 30(\mathrm{BR} \$ 380) /$ month $)^{(37)}$. Mother's education was categorised into five levels frequently used in Brazil (primary school not completed, primary school completed, secondary school not completed, secondary school completed and college degree).

\section{Statistical analysis}

There were three major parts of statistical analysis: descriptive statistics for the sample socio-demographic characteristics and the consumption of foods/beverages within meals and snacks, identification of dietary patterns and assessment of their relationship with the child's weight status.

Observed dietary patterns were described in terms of average consumption frequencies (ACF) for all dietary items, for each meal/snack presented in the food questionnaire. These are referred to as 'empirical dietary patterns' as opposed to 'discriminant dietary patterns', derived statistically in order to distinguish between weight status categories. A two-step procedure was used to find the latter: (a) selection of the variables with discriminant power regarding child overweight and obesity (sex and age adjusted BMI $z$-score $>+1)^{(35)}$ and (b) LCA of the selected variables to derive the dietary patterns of interest ${ }^{(38,39)}$.

Bivariate logistic regression for child overweight (including obesity) as binary variable was applied to all dietary items to select those with type I error significance level of $P<0.20$ in order to enhance the discriminant power of the latent classes regarding overweight. This variable selection was motivated by the objective of identifying the dietary patterns associated with overweight and obesity as opposed to the empirical patterns characterising overall eating behaviour. With twenty-one foods/ beverages over six meals/snacks, there were 126 binary variables indicating food intake. Many of the possible food combinations never took place and some were fairly rare $(<1 \%)$, leading to a highly singular food association matrix. Hence, a widely used criterion of variable selection for multivariate regression, based on bivariate regression significance level of $P<0 \cdot 20$, was applied to filter the dietary items for the LCA.

The optimal number of latent classes was determined by Lo-Mendell-Rubin criteria ${ }^{(39)}$. Successive LCA models were fitted with incremented number of latent classes until no improvement in model fit could be shown by the likelihood ratio test with a significance level of $P<0 \cdot 05$. Iteratively weighted least squares and logit transformation of binary dietary items were applied to identify distinct dietary patterns with well-separated clusters of individuals. LCA was repeated 300 times from random starting points to ensure its convergence to the global maximum. Posterior probability and its precision were calculated for consuming a dietary item on each eating occasion (conditional on the latent class membership) and divided by ACF. The latter can be considered an estimate of unconditional probability of consuming a dietary item for a given meal/snack, so that the ratio represents a deviation from the expected consumption frequency. This measure was denominated consumption frequency ratio (CFR) and its 95\% CI were calculated as normal deviates from the standard errors of the LCA posterior probability estimates.

The association between latent class membership and the independent variables (age, sex, school type, household monthly income level, mother's educational level, school shift) 
was initially explored by $\chi^{2}$ test. In addition, the association between the dietary patterns and child weight status was examined by regressing the latter onto latent class membership variable while adjusting for aforementioned independent and/ or confounding variables. The latter included mother's weight status, as there is a solid body of evidence on both genetic and environmental parental influence on children's dietary patterns ${ }^{(40)}$. Multinomial regression was used to calculate the probability of a child being non-overweight, overweight non-obese or obese for each latent class using non-overweight as the reference.

Statistical software Mplus version $6.04^{(39)}$ was used for LCA and Stata 12.0 (StataCorp LP, 2011) for logistic regression and descriptive statistics. Stratification, clustering and final population correction of the variance estimates were all taken into account as complex survey parameters in statistical analyses.

\section{Results}

The analysed sample included 1232 schoolchildren aged between 7 and 10 years with complete anthropometric and dietary recall data. The mean age was 9.1 (SD 0.8 ) years and $50.7 \%$ were boys. Table 1 presents key characteristics of the participants. The prevalence of children's overweight (including obese) was $34.4 \%$ and the prevalence of obesity was $10.5 \%$. A total of $63 \%$ of the children attended public schools. About $8 \%$ of the children were very poor, with monthly household income $<$ R $\$ 500$ ( $<270$ US\$) and additional $36.7 \%$ were poor, within the income range of R\$ 500-1500 (US\$ 270-807). The prevalence of mothers' overweight (including obese) was $26 \cdot 3 \%$. Just over $30 \%$ of the mothers attended college.

Table 2 shows the percentages of consumers by eating event, the percentage of dietary items consumed within each eating event and daily consumption frequency of food items. The mean values for total eating events frequency was 5.0 (SD 0.9) times/d, whereas those for meal and snack frequencies were $2 \cdot 8$ (SD 0.4) and $2 \cdot 2(\mathrm{SD} 0.9)$ times/d, respectively. There were no significant differences in the number of total eating events by sex $(P=0.52)$, type of school $(P=0.84)$ and children's weight status $(P=0.62)$. The same was true for the number of meals and snacks taken as separate outcomes (data not shown).

Lunch was reported by the majority of children (approximately 99\%), followed by breakfast (approximately 93\%) and dinner (approximately 92\%). Among the snacks, afternoon snack was the most frequent (approximately 89\%), followed by mid-morning (approximately 65\%) and evening snacks (approximately 63\%). The same top five foods were reported at lunch and dinner (rice, beef or poultry, beans, soft drinks and pasta), and at mid-morning, afternoon and evening snacks (fruits, sweets, soft drinks, bread and/or biscuits and chocolate milk), whereas the top five foods at breakfast were bread/biscuits, chocolate milk, coffee with milk, fruits and milk (Table 2).

Bivariate logistic regression found thirty-eight dietary items associated with child overweight (including obesity) using the previously established significance level of $P<0 \cdot 20$ to select the variables with discriminant power regarding child weight status for subsequent LCA (Table 3). The largest magnitude of OR was
Table 1. Characteristics of schoolchildren ( $n$ 1232) (Numbers and percentages)

\begin{tabular}{|c|c|c|}
\hline Characteristics & $n$ & $\%$ \\
\hline \multicolumn{3}{|l|}{ Age } \\
\hline $7.0-7.99$ & 276 & $22 \cdot 4$ \\
\hline $8.0-8.99$ & 287 & $23 \cdot 3$ \\
\hline $9.0-9.99$ & 349 & $28 \cdot 3$ \\
\hline $10 \cdot 0-10.99$ & 322 & $26 \cdot 1$ \\
\hline \multicolumn{3}{|l|}{ Sex } \\
\hline Boys & 625 & $50 \cdot 7$ \\
\hline Girls & 607 & $49 \cdot 3$ \\
\hline \multicolumn{3}{|l|}{ School type } \\
\hline Public & 782 & 63.5 \\
\hline Private & 450 & $36 \cdot 5$ \\
\hline \multicolumn{3}{|c|}{ Household monthly income level in $\mathrm{R} \$$ (minimum wage range) ${ }^{\star}$} \\
\hline $100-500(0.26-1.31)$ & 100 & 8.2 \\
\hline $501-1000(1.32-2.63)$ & 282 & 22.9 \\
\hline $1001-1500(2.64-3.95)$ & 170 & $13 \cdot 8$ \\
\hline $1501-2000(3.96-5 \cdot 26)$ & 113 & $9 \cdot 1$ \\
\hline $2001-2500(5 \cdot 27-6 \cdot 58)$ & 33 & $2 \cdot 7$ \\
\hline$>2500(>6.58)$ & 311 & $25 \cdot 2$ \\
\hline Missing & 223 & $18 \cdot 1$ \\
\hline \multicolumn{3}{|l|}{ Child weight status $\dagger$} \\
\hline Non-overweight & 809 & 65.6 \\
\hline Overweight non-obese & 294 & 23.9 \\
\hline Obese & 129 & 10.5 \\
\hline \multicolumn{3}{|l|}{ Mother's weight status $\ddagger$} \\
\hline Underweight & 50 & 4.1 \\
\hline Normal weight & 808 & $65 \cdot 6$ \\
\hline Overweight & 223 & $18 \cdot 1$ \\
\hline Obese & 101 & 8.2 \\
\hline Missing & 50 & 4.0 \\
\hline \multicolumn{3}{|l|}{ Mother's educational level } \\
\hline Primary school not completed & 216 & 17.5 \\
\hline Primary school completed & 103 & 8.3 \\
\hline Secondary school not completed & 122 & 9.9 \\
\hline Secondary school completed & 362 & 29.4 \\
\hline College & 399 & $32 \cdot 4$ \\
\hline Missing & 30 & 2.5 \\
\hline
\end{tabular}

* Each level of $\mathrm{R} \$ 500$ corresponded to 1.31 minimum wage at the time of the survey $\left(\right.$ minimum wage $=$ \$US 204.30 $(\text { \$BR 380 })^{(37)}$ monthly; September 2007 exchange rate: $\mathrm{US} \$=\mathrm{R} \$ 1.86$ )

$+\mathrm{WHO}^{(35)}$.

$\mp$ World Health Organization ${ }^{(36)}$.

found for rarely $(<1 \%$, i.e. ACF $<0 \cdot 01)$ consumed foods such as pasta and fish at breakfast, green leaves at mid-morning, cheese at lunch, vegetable soup in the afternoon and cooked vegetables in the evening. Some of these atypical reports showed the direction of association not expected according to other similar food surveys - for example, increased odds of overweight/obesity for the children reporting vegetable consumption (green leaves at mid-morning and vegetable soup in the afternoon). Another noticeable result was that the same food might have opposite direction of its association with overweight/obesity depending on the timing of its consumption. For example, drinking milk in the afternoon was associated with $64 \%$ reduction of the overweight/obesity odds as opposed to $175 \%$ increase of these odds when milk was consumed at dinner. Similarly, bread/biscuits consumed at mid-morning snack increased the odds by $51 \%$ but decreased the same odds by 21 and $33 \%$ when consumed at afternoon and evening snacks, respectively. In addition, cheese at breakfast increased the odds by $53 \%$ and reduced the odds by $72 \%$ if consumed at lunch. 
Table 2. Food and beverage intake assessed by the third version of the Previous Day Food Questionnaire, percentage of consumers by eating event and by food item in each eating event, and daily consumption frequency $(n$ 1232)

(Percentages and $95 \%$ confidence intervals)

\begin{tabular}{|c|c|c|c|c|c|c|c|c|c|c|c|c|c|}
\hline & \multicolumn{12}{|c|}{ Consumers/eating event } & \multirow{3}{*}{$\begin{array}{l}\text { Consumption frequency } \\
\text { (times/d) (mean) }\end{array}$} \\
\hline & \multicolumn{2}{|c|}{ Breakfast } & \multicolumn{2}{|c|}{ Mid-morning snack } & \multicolumn{2}{|c|}{ Lunch } & \multicolumn{2}{|c|}{ Afternoon snack } & \multicolumn{2}{|c|}{ Dinner } & \multicolumn{2}{|c|}{ Evening snack } & \\
\hline & $\%$ & $95 \% \mathrm{Cl}$ & $\%$ & $95 \% \mathrm{Cl}$ & $\%$ & $95 \% \mathrm{Cl}$ & $\%$ & $95 \% \mathrm{Cl}$ & $\%$ & $95 \% \mathrm{Cl}$ & $\%$ & $95 \% \mathrm{Cl}$ & \\
\hline Food/beverage & 92.6 & $90 \cdot 7,94.5$ & $64 \cdot 7$ & $59.8,69.9$ & 98.9 & $98.0,99.7$ & $89 \cdot 3$ & $86 \cdot 2,92 \cdot 3$ & 92.3 & $89 \cdot 6,95 \cdot 1$ & $62 \cdot 8$ & $58 \cdot 4,67 \cdot 2$ & \\
\hline Bread, biscuits & $64 \cdot 3$ & $58 \cdot 8,69.7$ & $10 \cdot 6$ & $7 \cdot 0,14 \cdot 1$ & 1.8 & $0.1,3.5$ & 38.2 & $31 \cdot 4,45 \cdot 1$ & 18.9 & $14 \cdot 4,23 \cdot 3$ & 10.4 & $9 \cdot 0,11 \cdot 8$ & 1.4 \\
\hline Chocolate milk & 48.0 & $41 \cdot 8,54.2$ & 8.4 & $6 \cdot 1,10 \cdot 8$ & 0.8 & $0.1,1.5$ & $21 \cdot 0$ & $17 \cdot 8,24 \cdot 3$ & $7 \cdot 1$ & $5 \cdot 2,9 \cdot 0$ & $12 \cdot 9$ & $9.9,15.9$ & 1.0 \\
\hline Coffee with milk & $27 \cdot 1$ & $22 \cdot 3,31 \cdot 9$ & $1 \cdot 8$ & $0 \cdot 6,3 \cdot 0$ & 0.2 & $0.0,0.6$ & 11.2 & $4 \cdot 7,17 \cdot 7$ & $6 \cdot 1$ & $2 \cdot 9,9.3$ & 4.9 & $3 \cdot 3,6 \cdot 4$ & 0.5 \\
\hline Milk & 11.5 & $9.5,13.5$ & 2.0 & $0.7,3.2$ & 0.0 & $0.0,0.1$ & 4.3 & $3.1,5.5$ & 2.4 & $1.4,3.4$ & $5 \cdot 2$ & $3.5,6.9$ & 0.3 \\
\hline Yogurt & 10.5 & $8 \cdot 4,12 \cdot 6$ & 5.4 & $3.5,7.3$ & 1.2 & $0.3,2 \cdot 1$ & $7 \cdot 0$ & $5 \cdot 2,8 \cdot 7$ & $2 \cdot 2$ & $1 \cdot 6,2 \cdot 9$ & $6 \cdot 2$ & $4 \cdot 3,8 \cdot 1$ & 0.3 \\
\hline Cheese & 11.2 & $8 \cdot 1,14 \cdot 2$ & 1.5 & $0.8,2 \cdot 2$ & 0.7 & $0.1,1.4$ & $5 \cdot 9$ & $3.7,8 \cdot 1$ & 4.3 & $2 \cdot 4,6 \cdot 2$ & 1.6 & $0.8,2.5$ & 0.3 \\
\hline Rice & 1.0 & $0.3,2 \cdot 1$ & $4 \cdot 1$ & $0.9,7.3$ & 65.3 & $61 \cdot 7,68.8$ & 3.2 & $0.8,5.6$ & $30 \cdot 1$ & $26 \cdot 3,34 \cdot 0$ & $2 \cdot 3$ & $1.1,3.5$ & 1.1 \\
\hline Beans & 1.1 & $0 \cdot 8,2 \cdot \cdot 1$ & $3 \cdot 1$ & $0.0,6.4$ & $52 \cdot 4$ & $46 \cdot 3,58 \cdot 5$ & $2 \cdot 2$ & $0.5,3.9$ & $22 \cdot 7$ & $18 \cdot 8,26 \cdot 5$ & 2.0 & $0.8,3 \cdot 2$ & 0.8 \\
\hline Pasta & 0.6 & $0.0,1.3$ & $2 \cdot 4$ & $1.0,3 \cdot 8$ & 33.9 & $30.8,37.0$ & 1.6 & $0.6,2 \cdot 7$ & 23.5 & $20 \cdot 8,26 \cdot 2$ & $1 \cdot 2$ & $0 \cdot 2,2 \cdot 1$ & 0.6 \\
\hline Beef, poultry & 1.8 & $0.8,2 \cdot 3$ & 3.5 & $1 \cdot 6,5 \cdot 4$ & 57.0 & $53.5,60 \cdot 4$ & $4 \cdot 1$ & $2 \cdot 0,6 \cdot 1$ & 28.4 & $25 \cdot 4,31.4$ & 2.5 & $1.2,3.9$ & 1.0 \\
\hline Fish, seafood & 0.8 & $0.0,1.7$ & 0.2 & $0.0,0.4$ & $10 \cdot 2$ & $7 \cdot 9,12.4$ & 0.2 & $0.0,0.4$ & 4.4 & $3 \cdot 0,5 \cdot 8$ & 0.2 & $0.0,0.4$ & 0.2 \\
\hline Leafy vegetables & 0.3 & $0.0,0.6$ & 0.3 & $0.0,0.7$ & 17.0 & $13 \cdot 8,20 \cdot 1$ & 0.8 & $0.1,1.4$ & $6 \cdot 1$ & $4.2,8 \cdot 0$ & 0.3 & $0.0,0.6$ & 0.2 \\
\hline Vegetable soup & 0.7 & $0.2,1 \cdot 3$ & 1.2 & $0 \cdot 2,2 \cdot 3$ & 2.9 & $1 \cdot 1,4 \cdot 7$ & 0.9 & $0.2,1.5$ & 7.6 & $4.3,10.9$ & 3.1 & $2 \cdot 3,4 \cdot 0$ & 0.2 \\
\hline Cooked vegetables & 0.3 & $0.0,0.6$ & 0.5 & $0.0,1.0$ & $16 \cdot 4$ & $14 \cdot 2,18 \cdot 6$ & 1.6 & $0.3,2.9$ & 7.7 & $6 \cdot 1,9 \cdot 3$ & 0.8 & $0.0,1.6$ & 0.3 \\
\hline Fruits & $18 \cdot 3$ & $15 \cdot 8,20 \cdot 8$ & $17 \cdot 3$ & $15 \cdot 4,19 \cdot 3$ & $7 \cdot 0$ & $4 \cdot 2,9 \cdot 8$ & $21 \cdot 6$ & $16 \cdot 7,26 \cdot 5$ & 5.8 & $3 \cdot 3,8 \cdot 2$ & $15 \cdot 0$ & $11 \cdot 3,18 \cdot 7$ & 0.9 \\
\hline Fruit juices & $10 \cdot 7$ & $8 \cdot 3,13 \cdot 0$ & $7 \cdot 0$ & $4 \cdot 4,9 \cdot 7$ & 28.4 & $22 \cdot 8,33.9$ & $12 \cdot 1$ & $7 \cdot 0,17 \cdot 2$ & $16 \cdot 8$ & $13 \cdot 5,20 \cdot 1$ & 6.9 & $5 \cdot 3,8 \cdot 6$ & 0.8 \\
\hline Soft drinks & 4.6 & $3 \cdot 2,5 \cdot 9$ & $14 \cdot 8$ & $12 \cdot 2,17 \cdot 4$ & 43.5 & $35 \cdot 6,51 \cdot 3$ & $21 \cdot 2$ & $15 \cdot 1,27 \cdot 2$ & $31 \cdot 1$ & $26 \cdot 9,35 \cdot 3$ & 9.4 & $7 \cdot 2,11 \cdot 6$ & 1.3 \\
\hline Sweets & 8.5 & $6 \cdot 3,10 \cdot 6$ & $15 \cdot 3$ & $12 \cdot 9,17 \cdot 6$ & $12 \cdot 2$ & $9 \cdot 4,15 \cdot 1$ & $27 \cdot 8$ & $22 \cdot 7,32 \cdot 9$ & 9.5 & $7 \cdot 3,11 \cdot 7$ & 11.6 & $9 \cdot 0,14 \cdot 3$ & 0.9 \\
\hline Savoury snacks & 1.1 & $0.3,1.9$ & 4.8 & $3 \cdot 1,6 \cdot 4$ & 0.6 & $0.0,1 \cdot 2$ & $7 \cdot 2$ & $4 \cdot 0,10 \cdot 4$ & 1.0 & $0.0,1.9$ & 3.2 & $2.4,3.9$ & 0.2 \\
\hline French fries & 1.2 & $0.2,2 \cdot 1$ & 1.7 & $0.7,2.7$ & $8 \cdot 3$ & $6 \cdot 2,10 \cdot 4$ & 3.6 & $1 \cdot 7,5.4$ & 6.7 & $4.3,9.2$ & 3.1 & $1.5,4.7$ & 0.3 \\
\hline Hamburger, pizza & $2 \cdot 2$ & $0.9,3.5$ & 5.4 & $3.5,7.4$ & $4 \cdot 1$ & $2 \cdot 3,6 \cdot 0$ & $7 \cdot 1$ & $3.7,10.5$ & 11.7 & $8 \cdot 4,15 \cdot 0$ & 3.3 & $1 \cdot 8,4 \cdot 8$ & 0.3 \\
\hline
\end{tabular}


Table 3. Discriminant dietary patterns identified by latent class analysis in six daily eating events

(Consumption frequency ratio (CFR) and $95 \%$ confidence intervals)

\begin{tabular}{|c|c|c|c|c|c|c|c|c|c|c|c|c|}
\hline \multirow[b]{3}{*}{ Meal/snack } & \multirow{3}{*}{$\begin{array}{l}\text { Foods/beverages discriminant } \\
\text { for overweight/obesity } \ddagger\end{array}$} & & & \multirow[b]{3}{*}{$\mathrm{ACF}$} & \multicolumn{8}{|c|}{ Latent classes (discriminant dietary patterns) $\dagger$} \\
\hline & & \multicolumn{2}{|c|}{ Bivariate logistic regression } & & \multicolumn{2}{|c|}{$A(32 \cdot 3 \%)$} & \multicolumn{2}{|c|}{ B $(48.6 \%)$} & \multicolumn{2}{|c|}{ C (15.1\%) } & \multicolumn{2}{|c|}{ D $(4.0 \%)$} \\
\hline & & OR & $P$ & & CFR & $95 \% \mathrm{Cl}$ & CFR & $95 \% \mathrm{Cl}$ & CFR & $95 \% \mathrm{Cl}$ & CFR & $95 \% \mathrm{Cl}$ \\
\hline \multirow{8}{*}{ Breakfast } & Yogurt & $1 \cdot 18$ & 0.19 & 0.105 & 1.56 & $0.01,3.11$ & 0.86 & $0.62,1.10$ & 0.41 & $0.00,1.49$ & $0.40^{\star}$ & $0.00,0.85$ \\
\hline & Cheese & 1.53 & 0.09 & 0.112 & 1.50 & $0.00,2.99$ & 0.74 & $0.48,1.01$ & 0.73 & $0.06,1.39$ & $1 \cdot 16$ & $0.55,1.78$ \\
\hline & Rice & 0.59 & 0.15 & 0.010 & 0.77 & $0.01,2.09$ & 0.77 & $0.01,1.71$ & 2.40 & $0.01,10 \cdot 70$ & $0.00^{*}$ & $0.00,0.00$ \\
\hline & Pizza/hamburger & $2 \cdot 87$ & 0.03 & 0.022 & 0.09 & $0.00,1.08$ & 1.74 & $0.84,2.64$ & 0.82 & $0.00,2 \cdot 71$ & $0.00^{\star}$ & $0.00,0.00$ \\
\hline & Pasta & 0.20 & 0.09 & 0.006 & 1.24 & $0.02,3.68$ & 0.93 & $0.02,2.15$ & 0.93 & $0.02,3.67$ & $0.00^{\star}$ & $0.00,0.00$ \\
\hline & Fish & 0.24 & 0.17 & 0.008 & $0.00^{*}$ & $0.00,0.00$ & 0.71 & $0.01,1.64$ & $4 \cdot 14$ & $0.01,18 \cdot 30$ & $0.00^{*}$ & $0.00,0.00$ \\
\hline & Meat & 0.22 & 0.12 & 0.018 & 0.28 & $0.01,1.05$ & $0.28^{*}$ & $0.01,0.61$ & 5.08 & $0.01,20.40$ & $0.00^{\star}$ & $0.00,0.00$ \\
\hline & Fruit juice & 1.68 & 0.09 & 0.106 & $1 \cdot 18$ & $0.25,2 \cdot 12$ & $0.73^{*}$ & $0.51,0.95$ & 1.69 & $0.00,5.45$ & $0 \cdot 15^{\star}$ & $0.00,0.50$ \\
\hline \multirow[t]{9}{*}{ Mid-morning snack } & Bread/biscuits & 1.51 & 0.10 & 0.106 & 1.21 & $0.06,2.36$ & 0.64 & $0.27,1.02$ & 1.96 & $0.00,6.44$ & $0.00^{*}$ & $0.00,0.00$ \\
\hline & Yogurt & 1.56 & 0.07 & 0.054 & 0.91 & $0.18,1.64$ & 0.85 & $0.38,1.33$ & 1.91 & $0.00,4.60$ & $0.00^{*}$ & $0.00,0.00$ \\
\hline & Rice & 1.41 & 0.18 & 0.041 & $0.00^{*}$ & $0.00,0.00$ & $0.12^{*}$ & $0.00,0.32$ & $0.00^{*}$ & $0.00,0.00$ & $23 \cdot 40^{*}$ & $21 \cdot 00,25 \cdot 80$ \\
\hline & Sweets & 0.50 & 0.02 & 0.153 & 0.98 & $0.32,1.63$ & 1.07 & $0.82,1.33$ & 0.97 & $0.05,1.90$ & $0.35^{\star}$ & $0.08,0.62$ \\
\hline & French fries & 1.92 & 0.20 & 0.017 & 0.53 & $0.01,2.02$ & 1.29 & $0.37,2 \cdot 20$ & 1.05 & $0.01,4.83$ & 0.94 & $0.01,3.00$ \\
\hline & Fruits & 1.73 & 0.01 & 0.173 & 0.84 & $0.21,1.48$ & 0.90 & $0.75,1.05$ & 1.91 & $0.00,3.93$ & $0.06^{\star}$ & $0.00,0.21$ \\
\hline & Beans & 1.59 & 0.13 & 0.031 & $0.00^{*}$ & $0.00,0.00$ & $0.19^{*}$ & $0.00,0.51$ & $0.00^{*}$ & $0.00,0.00$ & $22 \cdot 70^{*}$ & $11 \cdot 10,34 \cdot 30$ \\
\hline & Green leaves & $5 \cdot 26$ & 0.17 & 0.003 & $0.00^{*}$ & $0.00,0.00$ & 0.86 & $0.03,1.98$ & $0.00^{*}$ & $0.00,0.00$ & $15 \cdot 50^{*}$ & $2 \cdot 55,28.40$ \\
\hline & Vegetable soup & 0.39 & 0.20 & 0.012 & $0.16^{*}$ & $0.01,0.95$ & 0.89 & $0.10,1.67$ & 3.06 & $0.01,10.00$ & 1.29 & $0.01,4.28$ \\
\hline \multirow[t]{3}{*}{ Lunch } & Cheese & 0.28 & 0.15 & 0.007 & 0.54 & $0.01,1.88$ & 1.63 & $0.03,3.23$ & $0.00^{*}$ & $0.00,0.00$ & $0.00^{*}$ & $0.00,0.00$ \\
\hline & Rice & 0.82 & 0.12 & 0.653 & $1.51^{*}$ & $1.34,1.69$ & $0.49^{*}$ & $0.31,0.66$ & $1.47^{*}$ & $1.23,1.71$ & $1.36^{\star}$ & $1.24,1.48$ \\
\hline & Beans & 0.72 & 0.17 & 0.524 & $1.80^{*}$ & $1 \cdot 38,2 \cdot 22$ & $0.26^{*}$ & $0.00,0.52$ & $1.61^{*}$ & $1.28,1.94$ & 1.21 & $0.94,1.48$ \\
\hline \multirow[t]{7}{*}{ Afternoon snack } & Bread/biscuits & 0.79 & 0.06 & 0.382 & 0.68 & $0.00,2.68$ & 0.78 & $0.54,1.03$ & $2 \cdot 22^{*}$ & $1.75,2.69$ & $1.64^{\star}$ & $1 \cdot 18,2 \cdot 10$ \\
\hline & Chocolate milk & 0.80 & 0.13 & 0.210 & 0.48 & $0.00,2.91$ & 0.94 & $0.58,1.30$ & $2 \cdot 39^{*}$ & $1.58,3.20$ & 0.68 & $0.06,1.31$ \\
\hline & Milk & 0.36 & 0.01 & 0.043 & 0.33 & $0.00,1.10$ & 1.12 & $0.71,1.53$ & 1.98 & $0.00,6.04$ & 1.28 & $0.37,2.19$ \\
\hline & Soft drinks & 1.37 & 0.16 & 0.212 & 1.28 & $0.39,2.17$ & 0.97 & $0.72,1.22$ & 0.65 & $0.00,1.37$ & $0.46^{\star}$ & $0.19,0.73$ \\
\hline & Fruits & 0.76 & 0.11 & 0.216 & 1.21 & $0.72,1.71$ & $0.65^{*}$ & $0.39,0.91$ & 1.71 & $0.00,4.29$ & 0.84 & $0.60,1.09$ \\
\hline & Meat & 1.42 & 0.07 & 0.041 & 0.37 & $0.00,1.19$ & 0.94 & $0.45,1.42$ & 2.51 & $0.00,10 \cdot 70$ & 1.03 & $0.00,2.19$ \\
\hline & Vegetable soup & $2 \cdot 70$ & 0.08 & 0.009 & $0.00^{*}$ & $0.00,0.00$ & 1.34 & $0.25,2.44$ & 1.23 & $0.01,6 \cdot 28$ & 4.70 & $0.01,9.97$ \\
\hline \multirow[t]{6}{*}{ Dinner } & Milk & $2 \cdot 75$ & 0.06 & 0.024 & $1 \cdot 18$ & $0.00,2.91$ & $1 \cdot 18$ & $0.19,2.17$ & $0.25^{*}$ & $0.00,0.99$ & 0.25 & $0.00,1.57$ \\
\hline & Cheese & 1.94 & 0.04 & 0.043 & 1.70 & $0.29,3 \cdot 11$ & 0.86 & $0.50,1.23$ & 0.02 & $0.00,3.54$ & 0.63 & $0.00,1.68$ \\
\hline & French fries & 0.43 & 0.01 & 0.067 & 0.76 & $0.00,2.65$ & 1.07 & $0.75,1.39$ & $1 \cdot 10$ & $0.00,4.18$ & 1.68 & $0.72,2.64$ \\
\hline & Pizza/hamburger & 1.43 & 0.03 & 0.117 & 1.01 & $0.51,1.52$ & 1.24 & $0.89,1.59$ & 0.29 & $0.00,3.35$ & 0.65 & $0.26,1.03$ \\
\hline & Meat & $1 \cdot 15$ & 0.16 & 0.284 & $0.66^{*}$ & $0.32,0.99$ & 0.87 & $0.68,1.07$ & $2 \cdot 10$ & $0.00,6.66$ & $1 \cdot 18^{\star}$ & $1.06,1.29$ \\
\hline & Green leaves & 1.62 & 0.03 & 0.061 & $0.18^{*}$ & $0.00,0.66$ & 0.95 & $0.53,1.37$ & 2.72 & $0.00,9.40$ & 1.61 & $0.96,2.25$ \\
\hline \multirow{5}{*}{ Evening snack } & Bread/biscuits & 0.67 & 0.12 & 0.104 & 1.05 & $0.00,2.26$ & 0.66 & $0.30,1.01$ & $2 \cdot 13^{*}$ & $1.09,3.17$ & $0.52^{*}$ & $0.09,0.95$ \\
\hline & Chocolate milk & 0.57 & 0.13 & 0.129 & 0.91 & $0.53,1.29$ & 1.06 & $0.73,1.39$ & $1 \cdot 11$ & $0.01,2 \cdot 20$ & $0.54^{\star}$ & $0.19,0.89$ \\
\hline & Coffee and milk & 0.57 & 0.14 & 0.049 & 1.01 & $0.48,1.53$ & $0.37^{*}$ & $0.09,0.65$ & 2.68 & $0.00,7.16$ & $2 \cdot 33$ & $0.83,3.82$ \\
\hline & French fries & 0.45 & 0.20 & 0.031 & 1.07 & $0.00,2.27$ & 0.97 & $0.27,1.67$ & 1.07 & $0.00,3.98$ & 0.55 & $0.00,1.82$ \\
\hline & Cooked vegetables & 0.13 & 0.09 & 0.008 & $2 \cdot 18$ & $0.01,7.22$ & $0.39^{*}$ & $0.01,0.89$ & 0.41 & $0.00,1.49$ & $0.00^{*}$ & $0.00,0.00$ \\
\hline
\end{tabular}

$\mathrm{ACF}$, average consumption frequency; CFR, consumption frequency ratio (probability of food consumption given latent class membership/ACF); OR, OR for overweight (including obesity).

* Statistically significant $(P<0.05)$ CFR (keep in mind that 0.00 is rounded to the second decimal point and is always $>0$ ).

† Latent class (discriminant dietary patterns): A - 'higher food intake probability at lunch only'; B - 'lower food intake probability at all eating events'; C - 'higher food intake probability at lunch, afternoon and evening snacks'; and $D$ - 'lower food intake probability at breakfast and at the evening snack, higher food intake probability at other meals/snacks.

₹ Foods/beverages with $P<0.20$ in bivariate logistic regression. 
Four latent classes were identified as optimal solution considering the Lo-Mendel-Rubin criteria $^{(39)}$. These classes were labelled according to the time-of-day of eating events and the food intake probability (FIP).

The first latent class (A) comprised $32.3 \%$ of the children whose dietary pattern was labelled 'higher FIP at lunch only'. Their distinctive eating behaviour can be summarised as follows: compared with the sample average (ACF), they had significantly elevated probability of consuming rice $(51 \%)$ and beans ( $80 \%)$ at lunch but with significantly attenuated frequency of consuming selected foods at other times: no chance of consuming fish at breakfast; no consumption of rice, beans and green leaves, as well as $84 \%$ lower probability of eating vegetable soup at the mid-morning snack; no chance of eating vegetable soup at the afternoon snack; and 34 and $82 \%$ lower probability of eating meat and green leaves for dinner, respectively.

The second class (B) included $48.6 \%$ of the children, and their dietary pattern was labelled 'lower FIP at all eating events'. This class showed significantly lower probability of consuming selected foods for all daily meals/snacks when compared with the ACF: at breakfast ( 72 and $27 \%$ lower FIP for meat and fruit juice, respectively); at mid-morning snack (88 and $81 \%$ lower FIP for rice and beans, respectively); at lunch (51 and $74 \%$ lower FIP for rice and beans, respectively); at afternoon snack (35\% lower FIP for fruit intake); and at evening snack ( 63 and $61 \%$ lower FIP for coffee with milk and cooked vegetables, respectively). In addition, an important distinctive feature of this class was the reduced probability of consuming beans and rice at lunch.

The third class (C) included $15 \cdot 1 \%$ of the children whose dietary pattern was named 'higher FIP at lunch, afternoon and evening snacks'. Their eating behaviour was similar to the first class regarding lunch, with significantly elevated probability of consuming rice (47\%) and beans (61\%), compared with the sample average (ACF). They also had significantly elevated probability of having bread/biscuits (122\%) and chocolate milk (139\%) at the afternoon snack, and having bread/biscuits again in the evening (113\%). Another distinctive feature was virtually no consumption of rice, beans and green leaves during mid-morning snack, no cheese at lunch, as well as $75 \%$ lower probability of consuming milk at dinner. The fourth latent class (D) included $4.0 \%$ of the children, and their dietary pattern was named 'lower FIP at breakfast and at the evening snack, higher FIP at other meals/snacks'. Their distinctive eating behaviour was characterised by significantly higher probability of eating rice (23.4 times), beans (22.7 times) and green leaves (15.5 times) at mid-morning snack compared with ACF. Significantly higher probability of eating rice (36\%) was also found at lunch. Their afternoon snack was characterised by a $64 \%$ higher probability of consuming bread/biscuits and a 54\% lower probability of drinking soft drinks; at dinner, there was an $18 \%$ higher chance of eating meat; for the evening snack, there was no chance of eating cooked vegetables and significantly lower chances of consuming bread/biscuits (48\% less) and chocolate milk ( $46 \%$ less).

The relationship between the independent and/or confounding variables and latent class membership on the other hand was initially explored by $\chi^{2}$ test, which found no statistically significant differences regarding child age $(P=0 \cdot 24)$ and sex $(P=0 \cdot 48)$. Opposite results were found for school type $(P=0 \cdot 02)$, household monthly income $(P<0 \cdot 01)$ and mother's education level $(P<0 \cdot 01)$. In addition, there were no statistically significant differences between the latent dietary patterns and having breakfast $(P=0.59)$, lunch $(P=0.24)$ and dinner $(P=0 \cdot 14)$, but the opposite was true for having mid-morning $(P<0 \cdot 01)$, afternoon $(P<0.01)$ and evening snacks $(P<0.01)$.

All children from the latent class D studied at public schools, almost all (97\%) attended the school in the morning, over half ( $52 \%)$ of them fell within the two lowest income quintiles and $39 \%$ of them were born to mothers with no more than primary school education. When compared with other dietary patterns, class D children had higher public school attendance (100 v. 58-76\%), attended school more often in the morning (97 v. 42-51\%) and belonged more often to low-income families (39 v. 12-29\%). In addition, these children had the lowest percentage of evening snack intake and the highest percentage of mid-morning snack intake (Table 4).

The impact of the independent variables on child weight status was further investigated by multivariate multinomial regression and presented as fully adjusted marginal probabilities of overweight (non-obese) and obesity for each category (Table 5). Class B children with lower probability of frequency intake at all eating events had obesity risk of $13 \%$ (95\% CI 9, 17). However, class A children whose main meal of the day was lunch had only $6 \%(95 \%$ CI 3, 9) obesity risk a statistically significant reduction by more than a half. Boys had twice the girls' obesity risk (14 v. 7\%) and the difference was statistically significant. Children of obese mothers were significantly more likely (30\%) to be obese compared with children of non-obese mothers $(\leq 11 \%)$. If the mother was not overweight, the risk of overweight in her child was lower (21\%) compared with such risk if the mother was overweight (nonobese) (33\%) or obese (32\%). Neither child age, nor school type or household monthly income level or mother's educational level showed a statistically significant association with overweight/obesity.

\section{Discussion}

The present study showed that latent food patterns derived from specific eating events consumed according to the timeof-day are associated with the risk of obesity, independently of socio-economic and demographic factors, in a representative sample of 7-10-year-old-Brazilian schoolchildren. Class A dietary pattern with higher FIP at lunch presented lower obesity risk compared with class B pattern with lower FIP at all eating events. According to our analysis of discriminant DP, lunch was the main meal of the day for the class A members who presented higher FIP for rice and beans at lunch and had no other meal/snack with distinctive FIP. A distinctive characteristic of class B group was the lower probability of consuming rice and beans at lunch - a traditional Brazilian combination dish. These results are in line with those from other Brazilian studies in which the 'Traditional' dietary pattern, which relied mainly on rice and beans, was associated with a 
Table 4. Proportions of children with selected socio-demographic characteristics within each of four latent class dietary patterns $(n 1232)$ (Numbers and percentages)

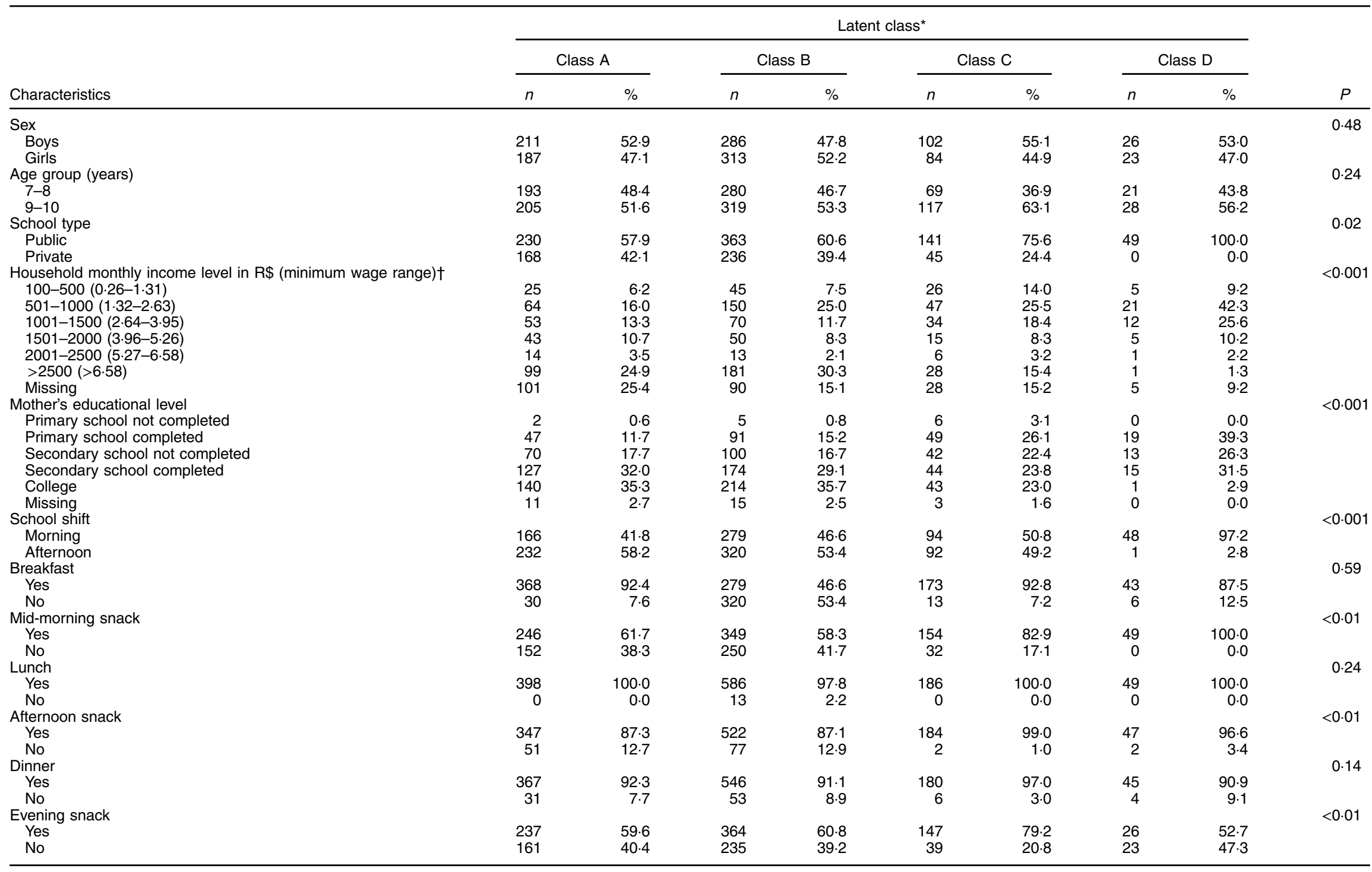

* Latent class: A - 'higher food intake probability at lunch only'; B - 'lower food intake probability at all eating events'; C - 'higher food intake probability at lunch, afternoon and evening snacks'; and D - 'lower food intake probability at

breakfast and at the evening snack, higher food intake probability at other meals/snacks.
† Each level of $\mathrm{R} \$ 500$ corresponded to 1.31 minimum wage at the time of the survey (minimum wage $=\$$ US 204.30 (\$BR 380) ${ }^{(37)}$ monthly; September 2007 exchange rate: US $\$=R \$ 1.86$ ). 
Table 5. The impact of independent variables on probability of child overweight and obesity adjusted in multivariate multinomial regression on child weight status (non-overweight used as reference)

(Mean values and $95 \%$ confidence intervals)

\begin{tabular}{|c|c|c|c|c|c|}
\hline \multirow[b]{3}{*}{ Independent variables } & \multirow[b]{3}{*}{$n$} & \multicolumn{4}{|c|}{ Marginal probability } \\
\hline & & \multicolumn{2}{|c|}{ Overweight } & \multicolumn{2}{|c|}{ Obesity } \\
\hline & & Mean & $95 \% \mathrm{Cl}$ & Mean & $95 \% \mathrm{Cl}$ \\
\hline \multicolumn{6}{|l|}{ Latent class of discriminant dietary patterns } \\
\hline A - 'higher FIP at lunch only' & 398 & 0.25 & $0.19,0.31$ & $0.06^{\mathrm{a}}$ & $0.03,0.09$ \\
\hline B - 'lower FIP at all eating events' & 599 & 0.24 & $0.20,0.28$ & $0 \cdot 13^{\mathrm{b}}$ & $0.09,0.17$ \\
\hline C - 'higher FIP at lunch, afternoon and evening snacks' & 186 & 0.18 & $0.14,0.23$ & 0.12 & $0.06,0.19$ \\
\hline D - 'lower FIP at breakfast and evening snacks, higher FIP at other meals/snacks' & 49 & 0.34 & $0.20,0.47$ & 0.11 & $0.05,0.17$ \\
\hline \multicolumn{6}{|l|}{ Child sex } \\
\hline Girls & 607 & 0.25 & $0.18,0.33$ & $0.07^{\mathrm{a}}$ & $0.05,0.09$ \\
\hline Boys & 625 & 0.23 & $0.19,0.26$ & $0.14^{\mathrm{b}}$ & $0.10,0.18$ \\
\hline \multicolumn{6}{|l|}{ Child age (years) } \\
\hline $7-8$ & 562 & 0.24 & $0.18,0.30$ & 0.11 & $0.07,0.15$ \\
\hline $9-10$ & 670 & 0.24 & $0.20,0.28$ & 0.10 & $0.05,0.15$ \\
\hline \multicolumn{6}{|l|}{ School type } \\
\hline Public & 782 & 0.23 & $0.17,0.30$ & 0.09 & $0.05,0.14$ \\
\hline Private & 450 & 0.25 & $0.17,0.31$ & 0.13 & $0.09,0.17$ \\
\hline \multicolumn{6}{|l|}{ Household monthly income level in $\mathrm{R} \$$ (minimum wage range) ${ }^{*}$} \\
\hline $100-500(0.26-1.31)$ & 100 & 0.24 & $0.18,0.30$ & 0.10 & $0.04,0.17$ \\
\hline $501-1000(1.32-2 \cdot 63)$ & 282 & 0.23 & $0.18,0.28$ & 0.10 & $0.05,0.14$ \\
\hline $1001-1500(2.64-3.95)$ & 170 & 0.22 & $0.13,0.31$ & 0.09 & $0.05,0.14$ \\
\hline $1501-2000(3.96-5 \cdot 26)$ & 113 & 0.15 & $0.10,0.20$ & 0.11 & $0.08,0.15$ \\
\hline $2001-2500(5 \cdot 27-6 \cdot 58)$ & 33 & 0.11 & $0.00,0.24$ & 0.07 & $0.01,0.13$ \\
\hline$>2500(>6.58)$ & 311 & 0.32 & $0.19,0.44$ & 0.11 & $0.03,0.18$ \\
\hline Missing & 223 & 0.23 & $0.15,0.30$ & 0.12 & $0.06,0.18$ \\
\hline \multicolumn{6}{|l|}{ Mother's educational level } \\
\hline Primary school not completed & 216 & 0.25 & $0.11,0.39$ & 0.08 & $0.00,0.21$ \\
\hline Primary school completed & 103 & 0.26 & $0.16,0.36$ & 0.10 & $0.03,0.17$ \\
\hline Secondary school not completed & 122 & 0.25 & $0.19,0.31$ & 0.12 & $0.06,0.18$ \\
\hline Secondary school completed & 362 & 0.23 & $0.17,0.29$ & 0.09 & $0.04,0.14$ \\
\hline College & 399 & 0.23 & $0.13,0.33$ & 0.11 & $0.06,0.17$ \\
\hline Missing & 30 & 0.24 & $0.09,0.39$ & $0 \cdot 10$ & $0.02,0.18$ \\
\hline \multicolumn{6}{|l|}{ Mother' weight status $†$} \\
\hline Non-overweight & 858 & $0.21^{a}$ & $0.16,0.26$ & $0.08^{\mathrm{a}}$ & $0.05,0.11$ \\
\hline Overweight & 223 & $0.33^{\mathrm{b}}$ & $0.27,0.38$ & 0.11 & $0.07,0.15$ \\
\hline Obese & 101 & 0.32 & $0.22,0.41$ & $0.30^{\mathrm{b}}$ & $0.19,0.40$ \\
\hline Missing & 50 & 0.13 & $0.03,0.23$ & 0.13 & $0.04,0.21$ \\
\hline
\end{tabular}

FIP, food intake probability.

${ }^{\mathrm{a}, \mathrm{b}}$ Mean values with unlike superscript letters were significantly different $(P<0.05)$ between the groups under comparison as indicated by non-overlapping $\mathrm{Cl}$.

${ }^{*}$ Each level of R $\$ 500$ corresponded to 1.31 minimum wage at the time of the survey (minimum wage $=\$ U S 204.30(\$ B R 380)^{(37)}$ monthly; September 2007 exchange rate: US $\$=R \$ 1.86)$.

† World Health Organization ${ }^{(36)}$

lower risk of overweight/obesity in both adolescents and $\operatorname{adults}^{(4,41)}$. They also agree with the findings from a US crosssectional survey, which indicated that the adults who consumed larger amount of the daily total energy intake at midday had lower risk of overweight/obesity ${ }^{(24)}$. Similarly, a Spanish population-based cohort showed that adults with a higher percentage of energy intake at lunch had a lower risk of weight gain $^{(26)}$. Other reports also suggested that the amount of energy or nutrients consumed at a certain time of the day influences subsequent eating occasions and is related to total daily energy intake in children $^{(17,20,21,27)}$ and adults ${ }^{(11,22-24,26,27)}$.

Higher obesity prevalence in children who reported lower FIP all over the day (class B) compared with other dietary patterns suggests under-reporting. This finding may be an example of 'reverse causality' and/or the reflection of factors such as social desirability of reporting a healthy diet, a well-known confounder in nutritional epidemiology ${ }^{(42)}$.
The school environment with adult supervision at the time of answering the questionnaire tends to enhance such behaviour. Despite the validity of PDFQ-3 at the group level ${ }^{(30)}$, it may not hold in obese children with a tendency to under-report. The sensitivity of LCA to detect such individuals and to provide clues for understanding an apparently counterintuitive relationship between dietary patterns and weight status reinforces the argument of its usability in nutritional epidemiology.

The time of the day of eating events influenced food choices and was an important distinguishing feature in the LCA-derived dietary patterns. For the $\mathrm{C}$ pattern, distinctive eating events occurred at afternoon and evening snacks (higher FIP for bread/ biscuits and chocolate milk), followed by lunch (higher FIP for rice and beans). Overall, these children showed a tendency to exceed the ACF of selected foods by at least $50 \%$ (CFR > 1.5) more often (eighteen of thirty-two foods) than the children in any other latent class $(5 / 32,2 / 32$ and $8 / 32$ for the classes A, B and D, 
respectively). Although not all of these tendencies were statistically significant and the $50 \%$ threshold is somewhat arbitrary, the frequency of consuming selected foods during the day was clearly elevated among the class $\mathrm{C}$ children. For the D pattern, however, distinctive FIP were spread between lower FIP at breakfast, higher FIP for rice, beans and green leaves at mid-morning snack, higher FIP for rice at lunch, followed by higher FIP for bread/biscuits at afternoon snack and higher FIP for meat/poultry at dinner. These children were mainly from poor families (Table 4) who may have compensated restricted food availability at home by consuming a hot meal with rice and beans, often provided by the public school at lunchtime, in the midmorning or in the afternoon. It is of notice that over twenty times more frequent consumption of rice and beans at mid-morning compared with ACF appears to be an anticipation of traditional Brazilian lunch after a low FIP at breakfast.

The comparison of the above results with other Brazilian studies is limited for several reasons. First, this is the first study that derived DP using LCA according to the time-of-day of eating events. Although precise timing was not recorded, the sequential nature of the events was clearly indicated for each meal or snack of the questionnaire and by the page order (online Supplementary Fig. S1). Second, there are many differences in the study design, age of participants, type of dietary assessment and statistical methods to derive DP. A nationally representative study used cluster analysis to identify three DP in 13-15-year-old adolescents evaluated by a FFQ: the 'Healthy' cluster with more frequent intake of healthy foods (cooked vegetables, fruits, milk, raw vegetables and beans) and less frequent intake of unhealthy foods (cookies, crackers, candies, sodas, fried snacks, cold cuts and French fries); the 'Unhealthy' cluster with reverse order of healthy and unhealthy foods; and the 'Mixed' cluster with similar frequencies of both healthy and unhealthy foods ${ }^{(3)}$. Another nationally representative study used factor analysis to derive DP in the Brazilian Household Budget Survey (2002-2003) and also identified three major DP: the 'Rice and beans' pattern was found in all regions; a 'Mixed pattern' including at least ten food groups was detected in the South, Southeast and Midwest region; and the 'Regional pattern' based on fish and nuts consumption was found in the Northern region ${ }^{(43)}$. Yet another study of this type conducted in 2008-2009 with individuals over 10 years of age verified the aggregation of DP among members of the same family and extracted three major DP: 'Traditional snack' (coffee, rolls, oils and fats, cheese), 'Traditional main meal' (rice, beans and other legumes, and meat) and 'Fast food snack' (sandwiches, processed meats, soft drinks, snacks, pizza) ${ }^{(40)}$. The traditional meal pattern compounded by rice and bean was also verified at lunch and dinner of adults from the Sao Paulo city ${ }^{(10)}$.

The present results corroborated the importance of rice and beans consumption identified in above studies and added the relevance of its timing for children's weight status. This combination dish has low energy density, high-quality plant protein, high carbohydrate and dietary fibre, micronutrients and B-vitamins ${ }^{(44)}$. In addition, dietary pulses (beans, lentils, chickpeas and dry peas) have a low glycaemic index ${ }^{(44)}$. Systematic reviews and meta-analysis of randomised controlled trials on adults confirmed the beneficial effect of dietary pulses in reducing body weight ${ }^{(45)}$ and increasing subjective satiety ${ }^{(46)}$.
An indirect indication of the benefits of consuming rice and beans at lunch has been the 3-fold increase in overweight in 5-9-year-old children between 1975 and 2009 (from 11 to 33\%) ${ }^{(47)}$, which coincided with a reduction in the consumption of rice, beans, milk, wheat and cassava flour, soyabean oil and sugar on one hand, and an accentuated increase in consumption of highly processed industrialised foods on the other hand ${ }^{(48)}$

Although LCA is a well-established statistical method for pattern analysis, its use for deriving dietary patterns has been surprisingly scarce $^{(6-8)}$. Major advantages of LCA in comparison with other statistical methods to derive DP include the following: (1) accounting for the (latent) sources of distinctive clustering of dietary items across groups of individuals, (2) not relying on highly implausible assumption of multivariate normality for often skewed distributions of dietary intake and (3) readily providing the probability of food intake for the dietary items analysed, thus facilitating the calculation of widely used effect size measures in nutritional epidemiology (e.g. risk ratio). LCA is unique in assuming that associations among the variables of interest may qualitatively differ across groups of individuals, which are unknown (latent) before the analysis is performed ${ }^{(49)}$. The within-group associations are shared by the group members but can vary between groups. In the context of FFQ, it is reasonable to assume systematic differences between individuals based on unknown food availability and taste preferences, which therefore act as latent classes to be inferred given the observed response. For example, those who highly value sweet taste are expected to have higher correlations between the frequencies of consuming dietary items with high sugar content (e.g. chocolate milk, cakes, biscuits, sugar-added beverages) compared with those who prefer savoury foods (e.g. rice and beans), given the availability of such foods. Of note, multilevel regression models do not account for this type of heterogeneity, as they assume an association pattern (covariance matrix) common to all individuals and random effects within group levels ${ }^{(50)}$. However, the groups must be known before the analysis ${ }^{(51)}$. Cluster analysis is akin to LCA but is usually applied to continuous variables (e.g. food weight) as opposed to binary indicators of food consumption during a meal/snack. The latter form is used in most FFQ and amenable to LCA, thus making it a method of choice for the analysis of dietary patterns based on the time-of-day of eating events.

Although the aim of the present study was to look for weight-status-discriminant dietary patterns, direct application of discriminant analysis was hampered by its assumption of multivariate normal distribution, which was clearly violated in the present study with a large number of binary food intake variables. Penalised regression methods ${ }^{(50)}$ such as reduced rank regression have been used ${ }^{(5)}$ to overcome this problem, but they do not account for the aforementioned latent heterogeneity. However, the logistic regression filtering of the LCA input variables with discriminant power regarding children's weight status performed a simplified version of penalisation by excluding less significant $(P>0.20)$ predictors. Although the latter is an ad boc statistical procedure to select the final set of independent variables in multivariate regression, it is widely used because it does not require specialised software and may provide a reasonable approximation for many practical purposes.

Among the limitations of the present study, the cross-sectional design is insufficient to infer causal relations between dietary 
patterns and child overweight/obesity, and a reverse causality bias between the two variables may have occurred for some children. Because of the dependence of the food choice on the time of the day, it was impossible to discern whether nutritional characteristics of a food or the timing of its intake or both caused the observed differences in the children's weight status. For example, rice and beans are part of a traditional Brazilian lunch and are rarely consumed at breakfast or at snacks. Second, questions about portion size, which must be known in order to assess energy intake, are not included in the questionnaire, and dietary information is derived from the children themselves (without help from parents or guardians) using the PDFQ-3. The PDFQ-3 was formulated in order to optimise recall by 7-10-year-old children of the intake of twenty-one food items in each of the six eating events on the previous day, ordered chronologically to allow meal timing analysis ${ }^{(30)}$. Because of the time constraints on its administration, only three main meals and three snacks could be illustrated to keep the questionnaire relatively brief and easy to complete with minimal assistance. The choice of focusing on food intake frequency instead of portion size was also made in other similar instruments in different countries ${ }^{(9)}$. In the age range of the present study (7-10 years), children spend considerable amount of time unsupervised by their parents, who therefore could not validate the children's dietary recall. The improvement of recall accuracy with age is well recognised ${ }^{(42)}$ and was demonstrated in the validation study with the PDFQ- $3^{(30)}$, where average sensitivity of dietary recall was $61.4 \%$ in 6-8-year-old and $76.6 \%$ in 9-11-year-old children. Third, the results obtained by LCA tend to be highly data driven and therefore require cross-validation with other independent samples.

By knowing the time-of-day and the physical and social environment in which an eating event takes place (e.g. in school $v$. at home), different types of dietary interventions may be warranted for children with different DP, according to their eating timing and the socio-demographic characteristics related to food availability. For example, healthy eating messages may be targeted to schoolchildren, their parents and school authorities, in order to encourage the consumption of rice and beans at lunch. In addition, adequate meal timing may improve public schools' menus in terms of balancing meal $v$. snack intake. Future studies should also include physical activity and sleep-wake duration data to assess their influence on circadian rhythms of food intake in children.

In conclusion, LCA identified four dietary patterns based on the time-of-day of eating events of selected dietary items and their associations with children's weight status. These patterns were also associated with school type, household monthly income, mother's educational level and school shift. Children having the traditional rice and beans lunch as the main meal of the day were least likely to be obese, thus reinforcing the importance of these foods for Brazilian diet. Food-based dietary guidelines and interventions to promote healthy eating should include advice on eating times in a strategy to prevent child obesity.

\section{Acknowledgements}

The authors gratefully acknowledge the children, parents and the school authorities for their participation in the study.
The survey was funded by the Brazilian National Council for Scientific and Technological Development - CNPq (grant no. 402322/2005-3) (E. K., grant no. 300436/2010-6), (M. A. A. d. A., grant no. 305148/2011-7). A. S. L. and D. B. L. received a fellowship from the Foundation of Research from Santa Catarina State and the Brazilian Federal Agency for the Improvement of Higher Education, respectively. The study funders had no role in the design, analysis or writing of this article.

E. K. participated in the concept, data analysis, interpretation and writing the manuscript; A. S. L., D. B. L. and F. B. assisted in the manuscript interpretation and writing the manuscript; M. A. A. d. A. developed the measurement instruments, participated in study design, supervised the study implementation and writing the manuscript. All authors read and critically reviewed the manuscript.

The authors declare no conflicts of interest.

\section{Supplementary material}

For supplementary material/s referred to in this article, please visit https://doi.org/10.1017/S0007114516004128

\section{References}

1. Kant AK (2004) Dietary patterns and health outcomes. J Am Diet Assoc 104, 615-635.

2. Ambrosini GL (2014) Childhood dietary patterns and later obesity: a review of the evidence. Proc Nutr Soc 73, 137-146.

3. Tavares LF, Castro IR, Levy RB, et al. (2014) Dietary patterns of Brazilian adolescents: results of the Brazilian National School-Based Health Survey (PeNSE). Cad Saude Publica 30, 2679-2690.

4. Rodrigues PR, Pereira RA, Cunha DB, et al. (2012) Factors associated with dietary patterns in adolescents: a school-based study in Cuiabá, Mato Grosso. Rev Bras Epidemiol 15, 662-674.

5. Ambrosini GL, Emmett PM, Northstone K, et al. (2014) Tracking a dietary pattern associated with increased adiposity in childhood and adolescence. Obesity (Silver Spring) 22, 458-465.

6. Huh J, Riggs NR, Spruijt-Metz D, et al. (2011) Identifying patterns of eating and physical activity in children: a latent class analysis of obesity risk. Obesity (Silver Spring) 19, 652-658.

7. Iannotti RJ \& Wang J (2013) Patterns of physical activity, sedentary behavior, and diet in U.S. adolescents. I Adolesc Health 53, 280-286.

8. Magee CA, Caputi P \& Iverson DC (2013) Patterns of health behaviours predict obesity in Australian children. J Paediatr Child Health 49, 291-296.

9. Pala V, Lissner L, Hebestreit A, et al. (2013) Dietary patterns and longitudinal change in body mass in European children: a follow-up study on the IDEFICS multicenter cohort. Eur J Clin Nutr 67, 1042-1049.

10. de Oliveira Santos R, Fisberg RM, Marchioni DM, et al. (2015) Dietary patterns for meals of Brazilian adults. Br J Nutr 114, 822-828.

11. Eicher-Miller HA, Khanna N, Boushey CJ, et al. (2016) Temporal dietary patterns derived among the adult participants of the National Health and Nutrition Examination Survey 1999-2004 are associated with diet quality. J Acad Nutr Diet 116, 283-291.

12. Murakami K \& Livingstone MB (2016) Decreasing the number of small eating occasions ( $<15 \%$ of total energy intake) regardless of the time of day may be important to improve diet quality but not adiposity: a cross-sectional study in British children and adolescents. Br J Nutr 115, 332-341. 
13. Murakami K \& Livingstone MB (2016) Associations between meal and snack frequency and overweight and abdominal obesity in US children and adolescents from National Health and Nutrition Examination Survey (NHANES) 2003-2012. Br J Nutr 115, 1819-1829.

14. Taillie LS, Afeiche MC, Eldridge AL, et al. (2015) Increased snacking and eating occasions are associated with higher energy intake among Mexican children aged 2-13 years. J Nutr 145, 2570-2577.

15. Llauradó E, Albar SA, Giralt M, et al. (2016) The effect of snacking and eating frequency on dietary quality in British adolescents. Eur J Nutr 55, 1789-1797.

16. Coulthard JD \& Pot GK (2016) The timing of the evening meal: how is this associated with weight status in UK children? $\mathrm{Br} J$ Nutr 115, 1616-1622.

17. Eng S, Wagstaff DA \& Kranz S (2009) Eating late in the evening is associated with childhood obesity in some age groups but not in all children: the relationship between time of consumption and body weight status in U.S. children. Int J Behav Nutr Phys Act 6, 27-34.

18. Chermont Prochnik Estima C, da Costa RS, Sichieri R, et al. (2009) Meal consumption patterns and anthropometric measurements in adolescents from a low socioeconomic neighborhood in the metropolitan area of Rio de Janeiro, Brazil. Appetite 52, 735-739.

19. Bellisle F (2014) Meals and snacking, diet quality and energy balance. Physiol Behav 134, 38-43.

20. Bellisle F, Rolland-Cachera MF, Deheeger M, et al. (1988) Obesity and food intake in children: evidence for a role of metabolic and/ or behavioral daily rhythms. Appetite 11, 111-118.

21. Thompson OM, Ballew C, Resnicow K, et al. (2006) Dietary pattern as a predictor of change in BMI z-score among girls. Int J Obes 30, 176-182.

22. de Castro JM (2009) When, how much and what foods are eaten are related to total daily food intake. Br J Nutr 102, 1228-1237.

23. Almoosawi S, Prynne CJ, Hardy R, et al. (2013) Time-of-day and nutrient composition of eating occasions: prospective association with the metabolic syndrome in the 1946 British birth cohort. Int J Obes (Lond) 37, 725-731.

24. Wang JB, Patterson RE, Ang A, et al. (2014) Timing of energy intake during the day is associated with the risk of obesity in adults. J Hum Nutr Diet 27, Suppl. 2, 255-262.

25. Garaulet M \& Gómez-Abellán P (2014) Timing of food intake and obesity: a novel association. Physiol Behav 134, $44-50$.

26. Hermengildo Y, López-García E, García-Esquinas E, et al. (2016) Distribution of energy intake throughout the day and weight gain: a population-based cohort study in Spain. $\mathrm{BrJ}$ Nutr 115, 2003-2010.

27. Almoosawi S, Vingeliene S, Karagounis LG, et al. (2016) Chrono-nutrition: a review of current evidence from observational studies on global trends in time-of-day of energy intake and its association with obesity. Proc Nutr Soc 75, 487-500.

28. Blondin SA, Anzman-Frasca S, Djang HC, et al. (2016) Breakfast consumption and adiposity among children and adolescents: an updated review of the literature. Pediatr Obes 11, 333-348.

29. Leal DB, de Assis MA, González-Chica DA, et al. (2015) Changes in total and central adiposity and body fat distribution among 7-10-year-old schoolchildren in Brazil. Public Health Nutr 18, 2105-2114.

30. Assis MA, Benedet J, Kerpel R, et al. (2009) Validation of the third version of the Previous Day Food Questionnaire (PDFQ-3) for 6-to-11-years-old schoolchildren. Cad Saude Publica 25, 1816-1826.

31. Assis MA, Calvo MC, Kupek E, et al. (2010) Qualitative analysis of the diet of a probabilistic sample of schoolchildren from Florianopolis, Santa Catarina State, Brazil, using the Previous Day Food Questionnaire. Cad Saude Publica 26, 1355-1365.

32. Brasil, Ministério da Saúde (2006) Guia alimentar para a população brasileira: promovendo a alimentação saudável (Dietary Guidelines for the Brazilian Population: Promoting Healthy Eating). Brasília: Secretaria de Atenção à Saúde. http://189.28.128.100/nutricao/docs/geral/guia_alimentar_ conteudo.pdf (accessed July 2016).

33. Lohman TG, Roche AF \& Martorell R (1988) Anthropometric Standardization Reference Manual. Champaign, IL: Human Kinetics Press.

34. Frainer DE, Adami F, Vasconcelos Fde A, et al. (2007) Standardization and reliability of anthropometric measurements for population surveys. Arch Latinoam Nutr 57, 335-342.

35. de Onis M, Onyango AW, Borghi E, et al. (2007) Development of a WHO growth reference for school-aged children and adolescents. Bull World Health Organ 85, 660-667.

36. World Health Organization (2000) Consultation on Obesity. Obesity: Preventing and Managing the Global Epidemic. WHO Technical Report Series, no. 894. Geneva: WHO.

37. Brasil, Ministério do Trabalho (2016) Evolução do salário mínimo 1940-2016 (Evolution of minimum wage 1940-2016). http://tra balho.gov.br/images/Documentos/SalarioMinimo/EVOLEISM 1940a2016.pdf (accessed October 2016).

38. Patterson BH, Dayton CM \& Graubard BI (2002) Latent class analysis of complex sample survey data: application to dietary data. J Am Stat Assoc 97, 721-742.

39. Asparouhov T \& Muthén B (2012) Using Mplus TECH1 and TECH14 to test the number of latent classes. Mplus Web Notes No. 14. http://www.statmodel.com/examples/webnote.shtml (acessed March 2016).

40. Massarani FA, Cunha DB, Muraro AP, et al. (2015) Familial aggregation and dietary patterns in the Brazilian population. Cad Saude Publica 31, 2535-2545.

41. Sichieri R (2002) Dietary patterns and their associations with obesity in the Brazilian city of Rio de Janeiro. Obes Res 10, 42-48.

42. Livingstone MB, Robson PJ \& Wallace JM (2004) Issues in dietary intake assessment of children and adolescents. $\mathrm{Br} \mathrm{J}$ Nutr 92, S213-S222.

43. Nascimento S, Barbosa FS, Sichieri R, et al. (2011) Dietary availability patterns of the Brazilian macro-regions. Nutr J 10, 79.

44. Mudryj AN, Yu N \& Aukema HM (2014) Nutritional and health benefits of pulses. Appl Physiol Nutr Metab 39, 1197-1204.

45. Kim SJ, de Souza RJ, Choo VL, et al. (2016) Effects of dietary pulse consumption on body weight: a systematic review and meta-analysis of randomized controlled trials. Am J Clin Nutr 103, 1213-1223.

46. Li SS, Kendall CW, de Souza RJ, et al. (2014) Dietary pulses, satiety, and food intake: a systematic review and meta-analysis of acute feeding trials. Obesity 22, 1773-1780.

47. Instituto Brasileiro de Geografia e Estatística (2010) National Household Budget Survey: 2008/2009. Anthropometry and Nutritional Status of Children, Adolescents and Adults in Brazil. Rio de Janeiro: IBGE.

48. Levy-Costa RB, Sichieri R, Pontes NS, et al. (2005) Household food availability in Brazil: distribution and trends (1974-2003). Rev Saude Publica 39, 530-540.

49. Muthén B \& Asparouhov T (2009) Multilevel regression mixture analysis. J R Statist Soc Series A 172, 639-657.

50. Hastie T, Tibshirani R \& Wainwright M (2015) Statistical Learning with Sparsity: The Lasso and Generalizations. Boca Raton, FL: CRC Press.

51. Masyn KE (2013) Latent class analysis and finite mixture modeling. In The Oxford Handbook of Quantitative Methods in Psychology vol. 2, pp. 551-611 [Todd D. Little, editor]. New York: Oxford University Press. 